\title{
Bone health in children with hemolytic anemia: does the pathogenesis of hemolysis determine the phenotype of bone alteration?
}

\author{
Michael Schündeln ${ }^{1}$, Sarah Goretzki ${ }^{1}$, Pia Hauffa ${ }^{1}$, Laura Marschke ${ }^{2}$, Regina Wieland ${ }^{1}$,
} Jens Bauer ${ }^{1}$, Berthold Hauffa ${ }^{2}$ \& Corinna Grasemann ${ }^{2}$

UK-Essen, Kinderklinik, ${ }^{1}$ Department of Pediatric Hematology and Oncology, ${ }^{2}$ Department of Pediatric Endocrinology, Essen, Germany.

Introduction: Chronic hemolytic anemia is a rare condition characterized by an abnormal breakdown of erythrocytes. Impaired bone health has been described in patients with sickle cell disease and thalassemia. Thus far it is unknown whether bone health in children with other forms of hemolytic anemias is affected aswell.

Aim and Design: To assess bone health in pediatric patients with different hemolytic anemias.

Methods and Patient characteristics: Patients ( $n=46,25$ female) were recruited year-round to avoid seasonal effects at followup visits at the Department of Pediatric Hematology and Oncology, Kinderklinik Essen. The study was approved by the ethics committee of the UK-Essen and written informed consent was obtained from patients and parents.

Patients had the following diagnoses: HBSS disease $(n=17)$, HBSC disease $(n=2)$, HBSbeta thalassemia $(n=1)$, beta thalassemia major $(n=6)$, beta thalassemia minor $(n=1)$, hereditary spherocytosis $(n=14)$, glucose-6phosphate deficiency $(n=2)$, paroxysmal nocturnal hemoglobinuria $(n=1)$, and hemolytic anemia of unknown origin $(n=2)$. For patient characteristics see Table 1.

Clinical and biochemical parameters of growth, puberty, bone turnover, and vitamin D metabolism were assessed. Bone pain, physical activity and calcium/vitamin D intake were assessed via a questionnaire.

\begin{tabular}{|llll} 
& $\begin{array}{l}\text { All patients } \\
(\mathrm{n}=46)\end{array}$ & $\begin{array}{l}\text { HbSS } \\
(\mathrm{n}=17)\end{array}$ & $\begin{array}{l}\text { Spherocytosis } \\
(\mathrm{n}=14)\end{array}$ \\
\hline Female/male & $25 / 21$ & $9 / 8$ & $7 / 7$ \\
Age (years) & $9.8 \pm 4.4$ & $9.3 \pm 4.6$ & $10.44 \pm 4.4$ \\
& $(1.1-18.4)$ & $(1.1-18.38)$ & $(2.5-17.9)$ \\
Pubic hair & $-0.19 \pm 0.89$ & $-0.16 \pm 0.93$ & $0.16 \pm 0.45$ \\
stage SDS & $(-2.24-1.2)$ & $(-1.73-1.2)$ & $(-0.64-0.87)$ \\
BMI SDS & $0.04 \pm 1.07$ & $0.01 \pm 1.34$ & $0.23 \pm 0.76$ \\
& $(-3.1-2-17)$ & $(-3.1-2.17)$ & $(-1.58-1.44)$ \\
Height SDS & $-0.26 \pm 1.06$ & $-0.05 \pm 1.0$ & $0.157 \pm 0.6$ \\
& $(-3.6-2.27)$ & $(-1.81-2.27)$ & $(-0.79-1.23)$ \\
LDH (U/1) & $427 \pm 209$ & $\mathbf{6 0 7} \pm 171$ & $\mathbf{2 8 9} \pm 47$ \\
& $(164-1008)$ & $(\mathbf{4 2 4 - 1 0 0 )}$ & $\mathbf{( 2 1 3 - 3 6 2 )}$ *** \\
Bili (mg/dl) & $2.6 \pm 1.7$ & $\mathbf{3 . 2 5} \pm \mathbf{1 . 1}$ & $\mathbf{2 . 9} \pm \mathbf{1 . 9}$ \\
& $(0.2-6.9)$ & $\mathbf{( 1 . 5 - 5 . 5 )}$ & $\mathbf{( 1 . 1 - 6 . 9 ) *}$ \\
Retic (0/00) & $160 \pm 112$ & $193 \pm 103.8$ & $150.2 \pm 110.3$ \\
& $(17-352)$ & $(43-352)$ & $(17-340)$
\end{tabular}

Table 1: Clinical characteristics and parameters of disease activity. Mean $\pm S D$, (range) are displayed. Statistically significant differences between the group of patients with sickle cell anemia and spherocytosis (results of t-test are indicated with asterisks ( $\left.{ }^{*}: P<0.05,{ }^{* * *}: P<0.001\right)$
Development of bone-score: Based on relevant biochemical markers of bone metabolism (Serum: 25-OH VD, PTH, SAP/ BAP. Urine-NTX/DPD) and presence/absence of bone pain a scoring system for bone health was developed. Each pathological finding was reflected with a score of 1 (maximum possible score: 7). A bone-score of 3 and above was defined as impaired bone health.

Data analyses: Linear regressions, Spearman correlation analyses, chi-square tests and ttests were performed with PRISM 6 for MAC OS X (La Jolla, CA, USA).

\begin{tabular}{|c|c|c|c|}
\hline & |l Patients & HbSS & Spherocytos \\
\hline $\begin{array}{l}25 \mathrm{OH} \text { VD }<20 \\
\mathrm{ng} / \mathrm{ml}\end{array}$ & $80.5 \%(33 / 41)$ & $5.7 \%(13 / 15)$ & 6 \\
\hline $\begin{array}{l}25 \mathrm{OH} \text { VD }<10 \\
\mathrm{ng} / \mathrm{ml}\end{array}$ & $50 \%(20 / 41)$ & $80 \%(12 / 15)$ & $0 \% * * *$ \\
\hline BAP/SAP $\uparrow$ & $11.6 \%(4 / 43)$ & $13 \%(2 / 15)$ & $0 \%(0 / 13)$ \\
\hline PTH $\uparrow$ & $22.5 \%(9 / 40)$ & $21.4 \%(3 / 14)$ & $15.3 \%(2 / 13)$ \\
\hline TX/DPD $\uparrow$ & $8.6 \%$ & $5.7 \%(2 / 12)$ & $0 \%(0 / 12)$ \\
\hline Back Pain & $32.4 \%(12 / 37)$ & $41.7 \%(5 / 12)$ & $15.3 \%(2 / 13)$ \\
\hline Knee Pain & $9.4 \%(7 / 36)$ & $18.2 \%(2 / 11)$ & 7.7. \% (1/13) \\
\hline Bone Score $\geq 3$ & $32.6 \%(15 / 46)$ & $41.2 \%(8 / 17)$ & $7.1 \%(1 / 14)$ \\
\hline
\end{tabular}

Table 2: Parameters used for the calculation of the Bone Score. Percentage and fraction (in brackets) of affected patients are displayed. Statistically significant differences between the group of sickle cell anemia and spherocytosis patients (chi-square test) are indicated with asterisks (***. $P<0.001)$

\section{Results:}

- $80 \%$ of the patients showed a $25-\mathrm{OH}$ Vitamin $D$ deficiency ( $<20 \mathrm{ng} / \mathrm{ml}$ ) and $50 \%$ a severe deficiency $(<10 \mathrm{ng} / \mathrm{ml})$. Patients with HbSS had higher rates of severe deficiency.

A Bone Score of $>3$ was found in $33 \%$ of the patients and more often in HbSS patients.

- Low vitamin-D status was associated with higher likelihood of back pain or knee pain during exercise (Fig.1).

- In patients with sickle cell anemia the Bone score was inversely correlated with the calcium to creatinine ratio (Fig. 2).

- The osteoblastic maker osteocalcin was lower in HbSS and spherocytosis patients compared to healthy controls (Fig. 3)

- RANKL/OPG ratio, as a marker of osteoclast activity, is higher in patients compared to healthy controls (Fig. 3)

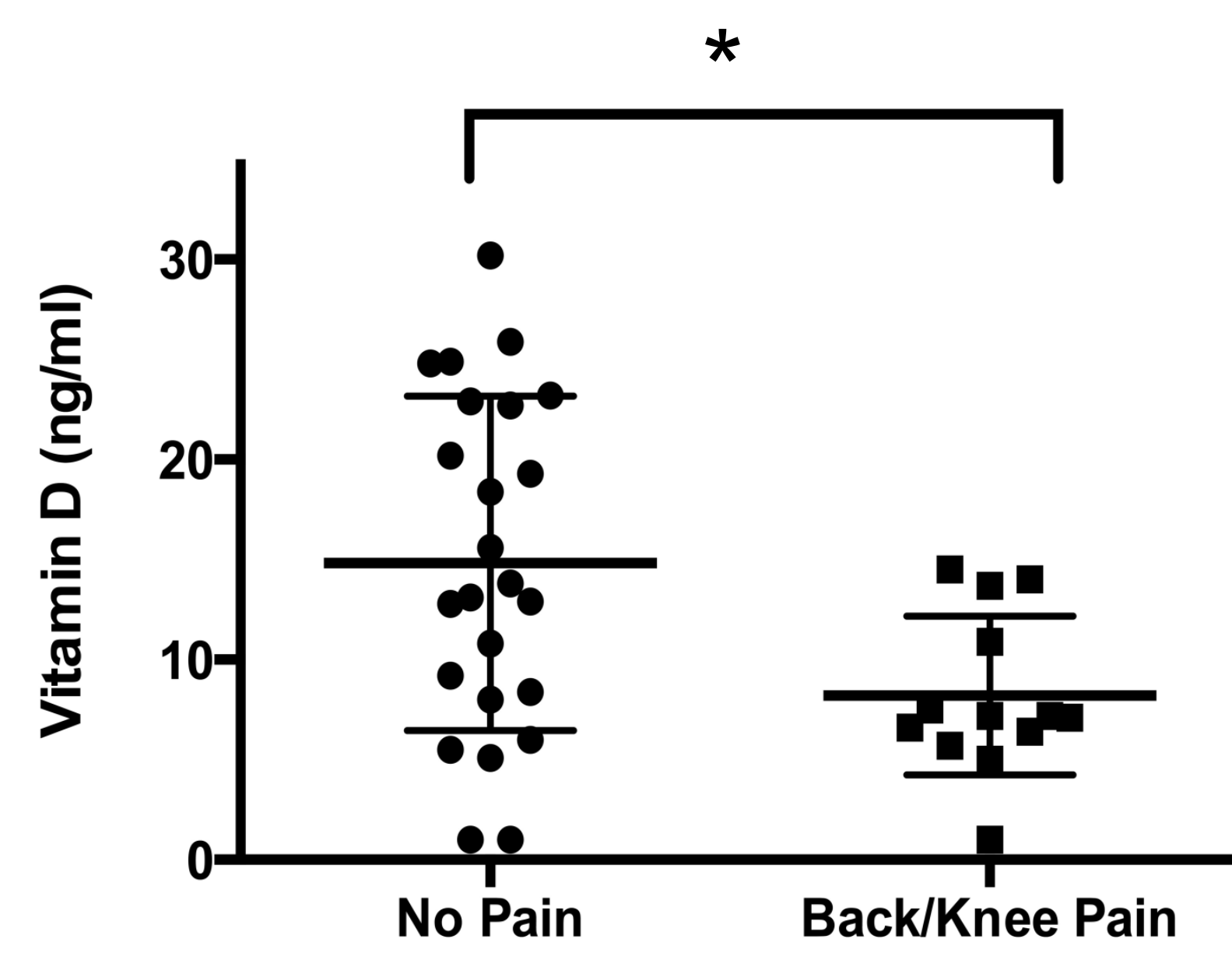

Figure 1: Association between pain (back pain and/or knee pain with exercise) and serum 25 $\mathrm{OH}$ vitamin $D$ levels. Statistically significant differences between the groups, determined via $t$-test, are indicated with asterisks (*: $P<0.05)$.
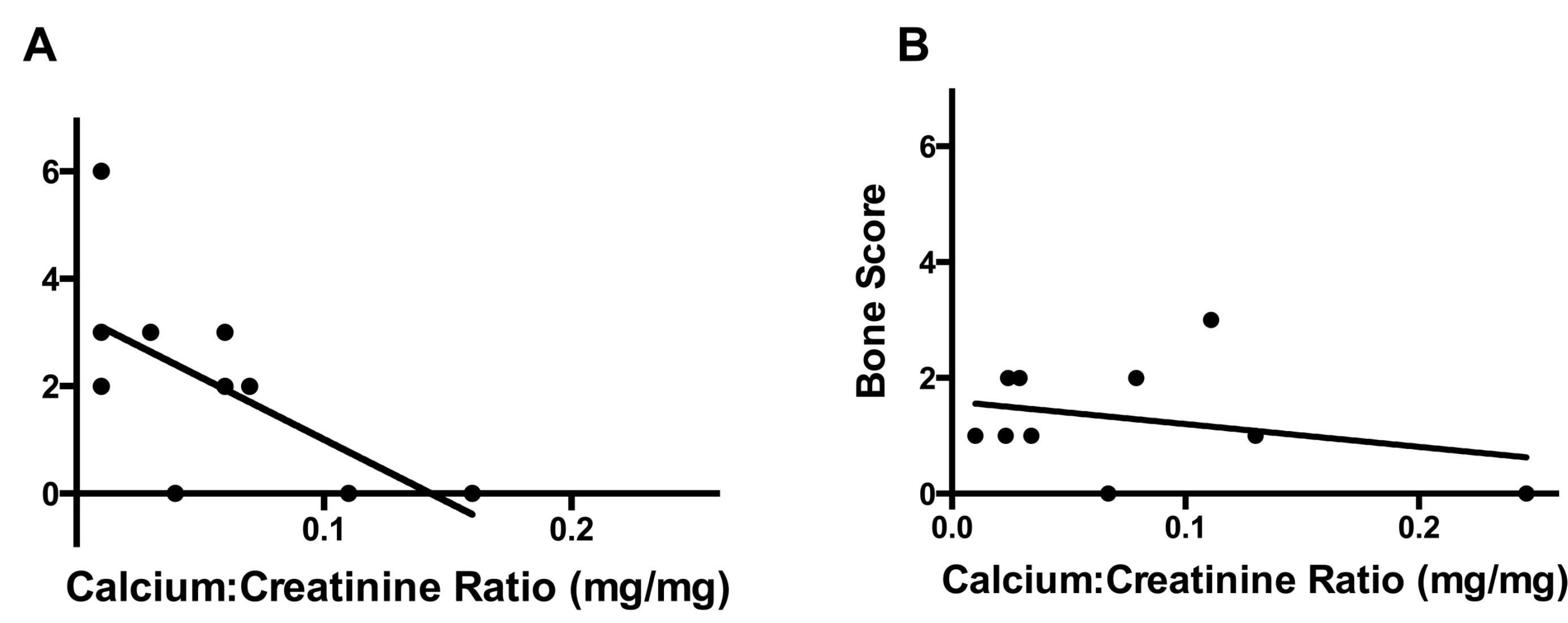

Fig 2: Bone score and calcium to creatinine ratio in urine displayed a significant negative correlation $(P=0.019, r$ -0.64) in patients with sickle cell anemia $(A)$ but not in patients with spherocytosis $(P=0.4, r=-0.29)(B)$. predicted values based on bivariate regression are indicated as solid lines.

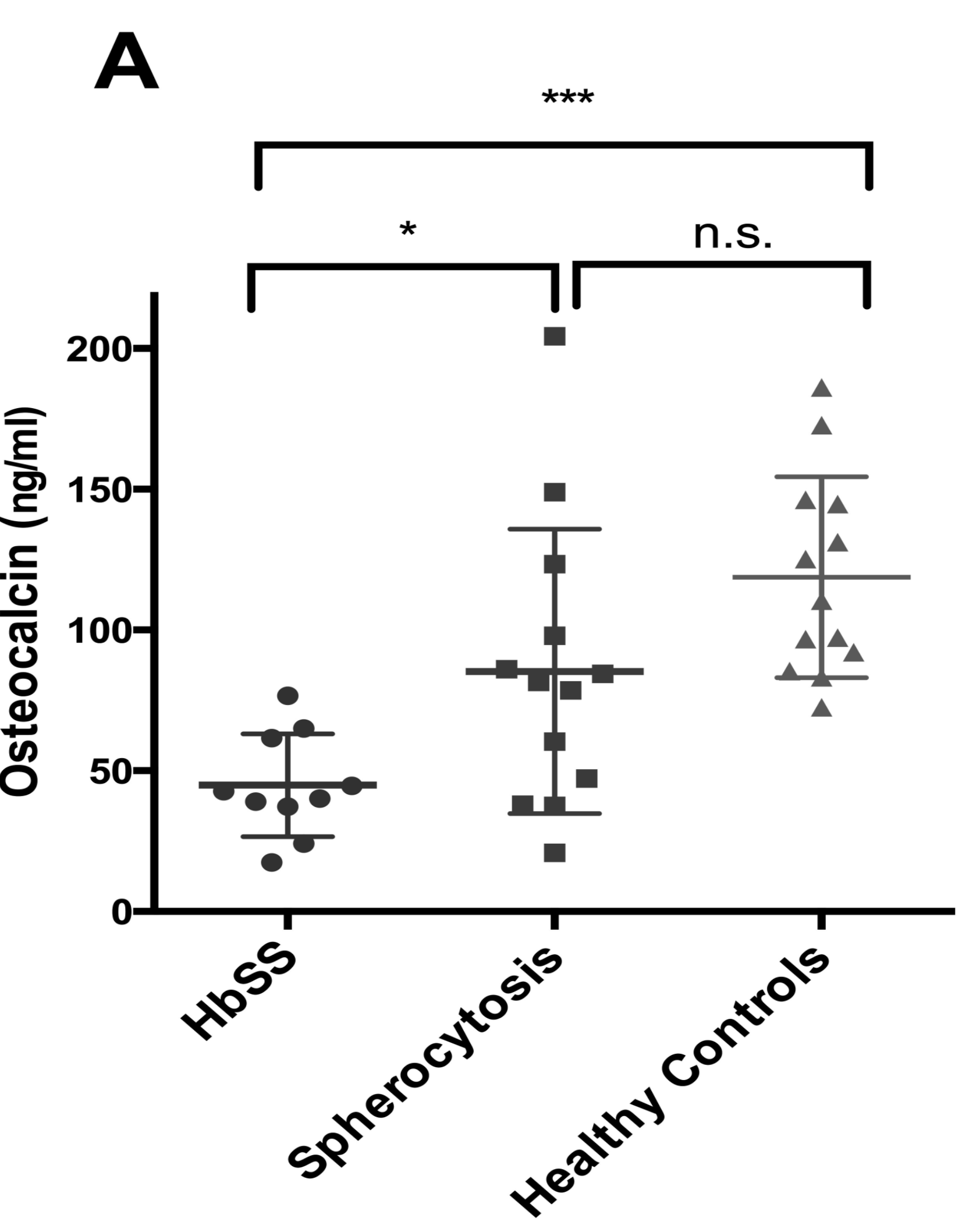

B

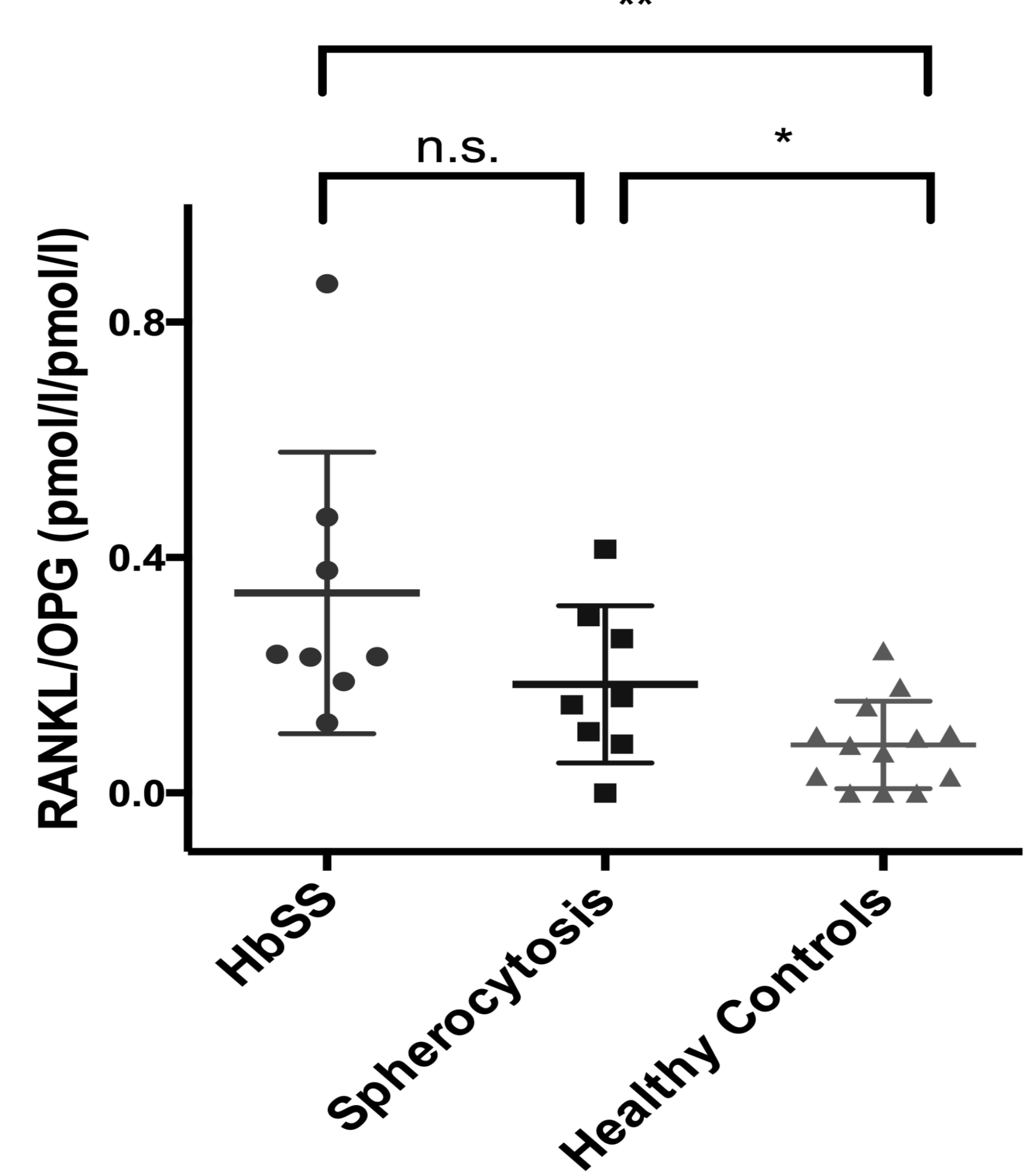

Figure 3: Parameters of bone remodeling in patients with sickle cell disease and spherocytosis compared with ageand sex-matched healthy controls. A) osteocalcin, B) RANKL OPG ratio. Statistically significant differences between the groups (t-test) are indicated with asterisks (*. $P<0.05$, * $P<0.01$, ***: $P<0.001)$

\section{Conclusions:}

Children with hemolytic anemias are at risk for impaired bone health

- the Bone Score is a useful tool to quantify the severity of bone impairment

patients with sickle cell disease display more severe alterations than patients with spherocytosis

- Low Calcium to creatinine ratio in sickle cell patients is associated with pathological Bone Scores

- Sickle cell disease and spherocytosis show distinct differences in osteocalcin and RANKL/OPG levels compared to healthy controls

- Patients should be monitored for sufficient vitamin D and calcium intake 